\title{
Major Obstacles to Implement a Full-Time Intensivist in Korean Adult ICUs: a Questionnaire Survey
}

\author{
Jun Wan Lee, M.D. ${ }^{1}$, Jae Young Moon, M.D., Ph.D. ${ }^{2}$, Seok Wha Youn, M.D., Ph.D. ${ }^{3}$, Yong Sup Shin, M.D., Ph.D. ${ }^{3}$, \\ Sang Il Park, M.D., Ph.D. ${ }^{3}$, Dong Chan Kim, M.D., Ph.D. , and Younsuk Koh, M.D., Ph.D. ${ }^{5}$ \\ ${ }^{1}$ Emergency ICU, Regional Emergency Center, ${ }^{2}$ Department of Internal Medicine, Division of Pulmonary and Critical Care Medicine, ${ }^{3}$ Department of Anesthesiology, Chungnam National \\ University Hospital, Daejeon; ${ }^{4}$ Department of Anesthesiology, Chonbuk National University Hospital, Jeonju; ${ }^{5}$ Division of Pulmonary and Critical Care Medicine, Department of Internal \\ Medicine, Asan Medical Center, Seoul, Korea
}

Background: Critical care physician staffing is a crucial element of the intensive care unit (ICU) organization, and is associated with better outcomes in ICUs. Adult ICUs in Korea have been suffering from inadequate full-time intensivists and nurses because of insufficient reimbursement rates ( $<50 \%$ of the original critical care cost) from the National Health Insurance System. Recently, full-time intensivists have been introduced as a prerequisite for adult ICUs of tertiary hospitals in Korea. The purpose of this study was to examine the perception of intensivist staffing among critical care program directors regarding the barriers and solutions when implementing an intensivist model of critical care in Korea.

Methods: An email survey of critical care program directors in designated teaching hospitals for critical care subspecialty training by the Korean Society of Critical Care Medicine was performed. The survey domains included vision, culture, resources, barriers, and potential solutions to implementing intensivist physician staffing (IPS).

Results: Forty-two critical care program directors were surveyed. A total of 28 directors $(66.7 \%)$ responded to email queries. Of these, 27 directors (96.4\%) agreed that IPS would improve the quality of care in the ICU, although half of them reported a negative perception of relevant clinical colleagues for the role of full-time intensivists and poor resources for IPS in their hospitals. Increased financial burden due to hiring full-time intensivists and concerns regarding exclusion from the management of their critically ill patients in the ICU, together with loss of income for primary attending physicians were stated by the respondents to be major barriers to implementing IPS. Financial incentives for the required cost from the health insurance system and enhancement of medical law relevant to critical care were regarded as solutions to these issues.

Conclusions: Critical care program directors believe that intensivist-led critical care can improve the outcome of ICUs. They indicated the financial burden due to IPS and underestimation of a full-time intensivist's role to be major barriers. The program directors agreed that a partnership between hospital leaders and the Ministry of Health and Welfare was needed to overcome these barriers.

Key Words: intensive care; intensive care unit; personnel staffing and scheduling.

\section{Introduction}

Although technological improvement for organ support and resuscitation is important, appropriate intensive care unit (ICU)

Received on January 26, 2016 Revised on March 29, 2016

Accepted on April 5, 2016

Correspondence to: Younsuk Koh, Department of Pulmonary and Critical Care Medicine, Asan Medical center, University of Ulsan College of Medicine,

88 Olympic-ro 43-gil, Songpa-gu, Seoul 05535, Korea

TEL: +82-2-3010-3134, FAX: +82-2-3010-4709

Email: yskoh@amc.seoul.kr

*No potential conflict of interest relevant to this article was reported. organization and management are also crucial for improved outcomes in the ICU.[1,2] Much evidence suggests that key elements of ICU organization-including appropriate intensivist physician staffing (IPS), nurse-patient ratio, a high volume of critically ill patients, and a multidisciplinary care team - are associated with improved quality of care in the ICU and better outcomes.[3-6] 
An intensivist is a certified critical care physician who provides clinical care exclusively in the ICU. The potential advantages of the presence of intensivists in ICUs have been reported; timely and frequent titration of therapy by being physically present in ICU, easier implementation of evidence-based protocols likely to benefit patients, enhanced communication and collaboration with other clinicians and medical specialists to provide optimum care, and the ability of the ICU manager to standardize care, discharge patients in a timely manner, and evaluate performance.[7] However, intensivist-staffing patterns in ICUs vary markedly. Although most European ICUs are fully staffed by intensivists, approximately one-third of ICUs in the USA are covered by an intensivist.[8] In Korea, a formal intensivist certification system was begun in 2009. The Korean Society of Critical Care Medicine (KSCCM) and the Korean government have made effort to improve the quality and safety of intensive care. A government regulation requiring intensivist staffing in tertiary hospitals in Korea has been in force since January 2015. As per the government regulation introduced, tertiary hospitals must have at least one full-time intensivist in the adult ICU. The intensivist is supposed to be present during the daytime and provide clinical care exclusively in the ICU, as suggested by the Leapfrog group IPS standards.[9] However, barriers to establishment of optimal IPS in adult ICUs of tertiary hospitals are expected under the current critical care medical environment in Korea. Furthermore, little information exists regarding the barriers related to IPS. To identify relevant barriers, we performed a questionnaire survey of critical care program directors regarding their perception of IPS.

\section{Materials and Methods}

\section{1) Questionnaire development}

We assessed five domains relevant to IPS implementation. These domains were based on a previously described framework for assessment of system change,[10] ICU director survey in the USA,[11] and expert opinions in Korea.[12] Domains included vision, culture, resources, barriers, and potential solutions for IPS. The questionnaire was formulated and modified by the members of the scientific committee of the KSCCM. Inquiries included the number of hospital beds, number of operating ICUs, ownership of the organization, accreditation as a tertiary hospital by the government, presence of a rapid response system or medical emergency team, knowledge of the requirements of IPS as per the government regulation, vision for improving quality of critical care by intensivist-led critical care, current hospital opinion surrounding IPS (individual hospital perception for intensivist staffing), current intensivists staffing, perceived resources, barriers and their potential solutions for IPS in their ICUs. The final survey contained 22 items comprising 2 items with a binary response (yes/no), 5 items with a descriptive response (numbers, additional comments), and 15 items with a Likert-scale response ranging from "strongly disagree" to "strongly agree."

\section{2) Survey administration and data collection}

The KSCCM has accredited 42 hospitals for the critical care program. Each hospital has a critical care program director with various background specialties, mainly anesthesiology and internal medicine. An e-mail survey was conducted by the scientific committee of KSCCM between March 2015 and April 2015.

\section{3) Statistical analysis}

We used the SPSS statistical software (version 19.0 K for windows; IBM, NY, USA) and computed the frequency and percentage of the responses to each question. Scaled responses are presented both continuously as mean and standard deviation (SD), and categorically as percentage in agreement. Responses both of positive ("agree" and "strongly agree") and negative ("disagree" and "strongly disagree") were grouped for the categorical analysis. Differences among respondents according to their characteristics including ownership of the institution, presence of a rapid response system, designation as a tertiary hospital, and hospital size were analyzed using the Kruskal-Wallis test.

\section{Results}

Of the 42 critical care program directors of KSCCM critical care training hospitals, 28 directors $(66.7 \%)$ responded to the e-mail survey. Organizational characteristics varied markedly among the responding hospitals. Table 1 lists 
the characteristics of the respondents, including hospital size, number of adult ICUs, ownership of the organization, presence of a rapid response system, and designation as a tertiary hospital. Most of the hospitals had 300-1000 beds (64.2\%), 1-5 adult ICUs (85.7\%), and operated privately $(75 \%)$. Only four hospitals $(14.3 \%)$ had a rapid response system for the acutely ill during their hospital stay. Twentyone hospitals $(75 \%)$ were accredited as tertiary hospitals at the time of the survey.

\section{1) Vision, culture, and resources for mandatory intensiv- ists staffing}

Table 2 shows the critical care program director's vision, culture regarding IPS in their own institution, and percep-

Table 1. Characteristics of 28 hospitals in the survey

\begin{tabular}{lc}
\hline Variable & Response (\%) \\
\hline Hospital size & \\
$300-1,000$ beds & $18(64.2)$ \\
$1,001-1,500$ beds & $5(17.8)$ \\
$>1,501$ beds & $5(17.8)$ \\
Number of adult ICU & \\
1 -5 units & $24(85.7)$ \\
$6-10$ units & $1(3.5)$ \\
$>11$ units & $3(10.7)$ \\
Ownership of hospital & \\
National & $7(25)$ \\
Private & $21(75)$ \\
Designation as tertiary hospital (yes) & $21(75)$ \\
Presence of rapid response system (yes) & $4(14.3)$ \\
\hline
\end{tabular}

ICU: intensive care unit. tion of resources for IPS implementation. Although organizational variation was evident, the vast majority of critical care program directors (96.4\%: percentage in agreement agree or strongly agree) agreed that mandatory intensivist staffing would improve the quality of critical care (rank: $4.4 \pm 0.6$ [mean $\pm \mathrm{SD}$ ] on a scale from 1 to 5 , with 1 indicating strong disagreement and 5 indicating strong agreement). Half of them perceived a positive culture for IPS, and agreed that there is a high expectation for intensivistled critical care in their own organizations (rank: $3.6 \pm 0.9$ [mean $\pm \mathrm{SD}$ ], \% in agreement: $50 \%$ ). However, they also had difficulties with mandatory IPS (rank: $3.1 \pm 1.1$ [mean \pm SD], \% in agreement: $42.9 \%$ ). Respondents reported scarce resources (intensivist availability and the financial burden of employment of intensivists) to implementing IPS (rank: $2.8 \pm 0.9$ [mean $\pm \mathrm{SD}$ ], \% in agreement to having rich resources: $28.5 \%$ ).

\section{2) Barriers and solutions}

In response to queries regarding barriers to IPS, the majority of the respondents replied "increased cost to hospital administration" as the major obstacle $(85.7 \%$ : \% in agreement), followed by primary attending physician's concerns regarding a decrease in their role in critical care and deterioration of their financial income $(57.1 \%$ and $50.0 \%$, respectively). Some of the critical care program directors $(42.9 \%)$ indicated difficulty in finding qualified intensivists.

To overcome these barriers, respondents considered financial incentive in terms of health-care reimbursement and legislation requiring IPS in all adult ICUs $(85.7 \%$, and $71.4 \%$, respectively) as potential solutions. Legal revi-

Table 2. Vision, culture, and perceived resources for intensivist staffing implementation among critical care program directors in Korea

\begin{tabular}{|c|c|c|}
\hline & Rank (mean $\pm \mathrm{SD}$ ) & $\begin{array}{l}\text { Percentage in agreement } \\
\text { (Strongly agree }+ \text { agree) }\end{array}$ \\
\hline \multicolumn{3}{|l|}{ Vision } \\
\hline Mandatory intensivist staffing will improve quality of critical care & $4.4 \pm 0.6$ & 96.4 \\
\hline \multicolumn{3}{|l|}{ Culture } \\
\hline High expectation for intensivist-led critical care in your hospital & $3.6 \pm 0.9$ & 50.0 \\
\hline Having trouble by revised law mandating intensivists staffing & $3.1 \pm 1.1$ & 42.9 \\
\hline \multicolumn{3}{|l|}{ Resources } \\
\hline Rich in intensivists, financial resources implementing intensivists in your hospital & $2.8 \pm 0.9$ & 28.5 \\
\hline
\end{tabular}

*On a scale from 1 to 5, with 1 indicating strong disagreement and 5 indicating strong agreement regarding vision of critical care program directors, current culture and resources.

SD: standard deviation. 
Table 3. Barriers and potential solutions for intensivist staffing implementation among critical care program directors in Korea

\begin{tabular}{lcc}
\hline & Rank (mean \pm SD) ${ }^{*}$ & $\begin{array}{c}\text { Percentage in agreement } \\
\text { (Strongly agree + agree) }\end{array}$ \\
\hline Barriers & $4.2 \pm 0.7$ & 85.7 \\
Increased cost to hospital administration & $3.5 \pm 0.9$ & 57.1 \\
Loss of control to certain physician groups & $3.4 \pm 1.2$ & 50.0 \\
Loss of income to certain physician groups & $3.3 \pm 0.9$ & 42.9 \\
Difficulty in finding qualified intensivists & & 85.7 \\
Potential solutions to barriers & $4.3 \pm 0.8$ & 71.4 \\
$\quad$ Financial incentives from government & $3.8 \pm 1.1$ & 39.2 \\
Legal revision mandating intensivists in all adult ICU & $3.0 \pm 1.1$ & 21.4 \\
Legal revision requiring closed ICUs in tertiary hospital & $2.3 \pm 1.0$ & \\
Public reporting of HSMR and risk adjusted ICU mortality & & \\
\hline
\end{tabular}

On a scale from 1 to 5 , with 1 indicating strong disagreement and 5 indicating strong agreement regarding barriers and potential solutions for intensivist staffing. SD: standard deviation; ICU: intensive care unit; HSMR: hospital standardized mortality ratio.

Table 4. Differences in perception on intensivist staffing according to hospital size

\begin{tabular}{|c|c|c|c|c|}
\hline \multirow{3}{*}{ Domain } & \multicolumn{4}{|c|}{ Beds } \\
\hline & $>1,501(n=5)$ & $1,001-1,500(n=5)$ & $<1,000(n=18)$ & $\mathrm{p}$-value \\
\hline & \multicolumn{4}{|c|}{ Number of respondents (Agree / strongly agree) } \\
\hline \multicolumn{5}{|l|}{ Vision } \\
\hline Mandatory intensivist staffing will improve quality of critical care & $1 / 4$ & $3 / 2$ & $10 / 7$ & 0.534 \\
\hline \multicolumn{5}{|l|}{ Culture } \\
\hline High expectation for intensivist-led critical care in your hospital & $1 / 4$ & $2 / 1$ & $5 / 1$ & $0.023^{*}$ \\
\hline Having trouble by revised law mandating intensivists staffing & $2 / 0$ & $2 / 1$ & $6 / 1$ & 0.485 \\
\hline \multicolumn{5}{|l|}{ Resources } \\
\hline Rich in intensivists, financial resources implementing intensivists in your hospital & $4 / 0$ & $1 / 0$ & $2 / 1$ & $0.025^{*}$ \\
\hline \multicolumn{5}{|l|}{ Barriers } \\
\hline Increased cost to hospital administration & $2 / 3$ & $5 / 0$ & $6 / 8$ & 0.194 \\
\hline Loss of control to certain physician groups & $2 / 0$ & $2 / 1$ & $10 / 2$ & 0.348 \\
\hline Loss of income to certain physician groups & $2 / 0$ & $2 / 2$ & $6 / 3$ & 0.08 \\
\hline Difficulty in finding qualified intensivists & $1 / 0$ & $2 / 1$ & $7 / 1$ & 0.717 \\
\hline \multicolumn{5}{|l|}{ Potential solutions to barriers } \\
\hline Financial incentives from government & $0 / 5$ & $3 / 2$ & $5 / 9$ & 0.285 \\
\hline Legal revision mandating intensivists in all adult ICU & $3 / 2$ & $2 / 2$ & $4 / 7$ & 0.76 \\
\hline Legal revision requiring closed ICUs in tertiary hospital & $1 / 0$ & $3 / 0$ & $6 / 1$ & 0.642 \\
\hline Public reporting of HSMR and risk adjusted ICU mortality & $0 / 0$ & $0 / 2$ & $4 / 4$ & 0.467 \\
\hline
\end{tabular}

*Statistically significant.

HSMR: hospital standardized mortality ratio, ICU: intensive care unit.

sion requiring closed ICUs in tertiary hospitals and public reporting of hospital standardized mortality ratio (HSMR) and/or risk-adjusted ICU mortality were perceived as lessimportant solutions (Table 3).

\section{3) Differences among respondents' perceptions}

In a subgroup analysis according to the respondents' characteristics (Table 4) —including ownership of the institution, presence of a rapid response system, designation as a 
tertiary hospital, and hospital size - only hospital size was associated with positive culture for IPS and perceived resources $(\mathrm{p}=0.023$ and 0.025 , respectively). Hospitals with larger beds ( $>1,000$ beds) had more frequently adopted a rapid response system $(\mathrm{p}=0.019)$.

\section{Discussion}

This study highlights the obstacles to optimal IPS under current medical practice in Korean adult ICUs. Although the overwhelming majority of critical care program directors in Korea believed that intensivists could improve the quality of critical care, they had both positive and negative opinions of intensivist staffing. Moreover, perceived current resources for optimal IPS seemed to be inadequate.

A previous study reported several obstacles to IPS in the USA.[11] Critical care program directors in Korea shared similar barriers to those in the USA in terms of structural and cultural challenges. However, the perceived barriers were somewhat different. The critical care program directors in Korea believed that the most significant barrier to IPS is the associated financial burden, followed by the concern of certain physician groups regarding loss of control of management of their patients in ICUs, whereas a loss of control of patients to certain physician groups in USA.[11]

As expected, the critical care program directors' concern is based on our medical cost reimbursement system. Korea has a compulsory National Health Insurance (NHI) system. Thus, all health care services are mandatory NHI providers under the National Health Insurance Act. All services, including hospital and physician costs, are paid on a fee-forservice basis. A crucial problem in critical care delivery is that the reimbursement rate for critical care was $\sim 46 \%$ of the actual expenditure in a 2004 NHI study.[12] Thus, hospital administrators were not motivated to adopt the intensivist model of critical care. Under the current payment system in Korea, an intensivist without general ward designation, outpatient clinic, or operation theatre responsibilities may not be able to make a profit for their institution. However, intensivist-led critical care can be cost saving by avoiding unnecessary admission to ICU, decreasing readmission after discharge and enhancing the efficiency of use of finite resources.[13]
Specialists working in hospitals are employed and paid using a performance based salary system in many hospitals, including academic hospitals, in Korea. About $74.2 \%$ of hospitals in Korea have introduced a performance based salary system.[14] Performance is determined by the volume of services provided by each specialist. Therefore, the attending physicians might be reluctant to relinquish control over their patient's care in the ICU, which indirectly is an important source of income.[11] Due to the performance based salary system on a fee for services basis, admitting physicians tend to be resistant to hand over control of patients, which is also an important barrier to IPS adoption.

The respondents considered to overcome these barriers by appropriate reimbursement of required medical costs together with legislation requiring intensivists in adult ICUs in Korea. At present, legal regulation of intensive care is truly far below the global standard.[12] In contrast to neonatal ICUs, there is no obligation to have a full-time intensivist in adult ICUs, as indicated in a medical regulation (enforcement rule article 28, paragraph 8 ). Of the critical care program directors, $71.4 \%$ agreed legal revision to be a potential solution to issues preventing IPS implementation.

The solutions suggested by the respondents should be addressed to enhance critical care in our society, because a qualified intensivist, defined as a physician certified in critical care medicine, is a crucial element of the organizational structure of the ICU. The current requirement for at least one intensivist for tertiary hospitals should be urgently revised, because intensivist/patient ratios impact patient care, education of trainees, and wellbeing of staff.[15] To relieve conflicts between general physicians and intensivists in the same hospital, the role of intensivists should be clearly defined in Korean hospitals by legislation regarding critical care. Mandating semi-closed ICU or closed ICU can be one of the legislative mean to clarify the role of intensivists. Regular auditing of ICU care performance through the Health Insurance Review and Assessment Service will facilitate optimal IPS.

In this study, most respondents did not agree with public reporting of the HSMR and severity adjusted ICU mortality as a solution. Public reporting and performance-based pay have been considered potential solutions to the crisis in quality in US health care, despite conflicting results.[16-19] While an assessment of the performance of each hospital 
and ICU in Korea is currently underway, further valid tools for assessing quality of care are required.

Despite these barriers, similar to a previous US study,[20] some organizations are performing intensivist-led critical care. These "pioneers" have demonstrated the benefits of the intensivist model of critical care during the H1N1 pandemic, severe sepsis, major trauma, and surgical critical care in Korea.[21-24] These motivated intensivists and institutions have generated support for system-level change in critical care in Korea. In our study, the expectations of intensivist-led critical care and perceived resources differed according to hospital size, although all physicians stated that intensivist-led critical care would improve the overall quality of intensive care. For wider adoption of intensivist-led critical care and improvement of the quality of critical care nationwide, more support for smaller hospitals is needed.

In a recent OECD health-care quality review,[25] Korea faces looming challenges — an ageing population, growing chronic disease burden and rising medical costs. Concerns regarding quality of care and patient safety are growing, necessitating a strengthened focus on governance. Intensivist staffing in ICUs is a potential solution to these challenges. Intensivists, hospital leaders, and policy makers should build the best model of critical care and disseminate it across the Korean health system.

This study has several limitations that might impact interpretation of the data. First, our sample size was small and the response rate was $66.7 \%$, which limited generalization of findings. However, the nation has 42 critical care program directors, and their overall response rate was similar to surveys of physician groups.[26] Second, we did not survey other stakeholders in the IPS, including hospital administrators, non-intensivist physicians, and policymakers. We believe that critical care program directors have seniority and clinical leadership in their organization; therefore, they have the greatest insight into the barriers and potential solutions under the current circumstances in Korea. Third, ICU staffing may depend on ICU characteristics, which necessitates an individual approach including alternative staffing models.[27] Further research is needed to develop the optimum model for each hospital.

In conclusion, there are several obstacles to intensivist physician staffing in Korean ICUs. An intensivist is a key player in an ICU organization, who should provide best practice on the frontline. Introduction of a sustainable and solid structure of intensivist staffing requires partnership among hospital leaders, policy makers, and physicians. Further research is warranted to define the best model of critical care delivery, which will further improve outcomes among the critically ill in Korea.

\section{Acknowledgements}

We thank the critical care program directors of the subjected ICUs for their participation in survey administration. This study was conducted under the auspices of the Korean Society of Critical Care Medicine, and was presented at the Critical Care Symposium of the Korean Society of Critical Care Medicine (KSCCM) on April 25, 2015 in Seoul, Korea.

\section{ORCID}

$\begin{array}{ll}\text { Jun Wan Lee } & \text { http://orcid.org/0000-0003-4630-597X } \\ \text { Jae Young Moon } & \text { http://orcid.org/0000-0001-8724-6289 } \\ \text { Sang II Park } & \text { http://orcid.org/0000-0002-2026-6848 } \\ \text { Dong Chan Kim } & \text { http://orcid.org/0000-0002-6881-126X } \\ \text { Younsuk Koh } & \text { http://orcid.org/0000-0001-5066-2027 }\end{array}$

\section{References}

1) Carmel S, Rowan K: Variation in intensive care unit outcomes: a search for the evidence on organizational factors. Curr Opin Crit Care 2001; 7: 284-96.

2) Nguyen YL, Wunsch H, Angus DC: Critical care: the impact of organization and management on outcomes. Curr Opin Crit Care 2010; 16: 487-92.

3) Pronovost PJ, Angus DC, Dorman T, Robinson KA, Dremsizov TT, Young TL: Physician staffing patterns and clinical outcomes in critically ill patients: a systemic review. JAMA 2002; 288: 2151-62.

4) Tarnow-Mordi WO, Hau C, Warden A, Shearer AJ: Hospital mortality in relation to staff workload: a 4-year study in an adult intensive-care unit. Lancet 2000; 356: 185-9. 
5) Kahn JM, Goss CH, Heagerty PJ, Kramer AA, O’Brien CR, Rubenfeld GD: Hospital volume and the outcomes of mechanical ventilation. N Engl J Med 2006; 355: 4150 .

6) Leape LL, Cullen DJ, Clapp MD, Burdick E, Demonaco HJ, Erickson JI, et al: Pharmacist participation on physician rounds and adverse drug events in the intensive care unit. JAMA 1999; 282: 267-70.

7) Pronovost PJ, Holzmueller CG, Clattenburg L, Berenholtz S, Martinez EA, Paz JR, et al: Team care: beyond open and closed intensive care units. Curr Opin Crit Care 2006; 12: 604-8.

8) Angus DC, Shorr AF, White A, Dremsizov TT, Schmitz RJ, Kelley MA, et al: Critical care delivery in the United States: distribution of services and compliance with Leapfrog recommendations. Crit Care Med 2006; 34:1016-24.

9) Gasperino J: The Leapfrog initiative for intensive care unit physician staffing and its impact on intensive care unit performance: a narrative review. Health Policy 2011; 102: 223-8.

10) Villa RA, Thousand JS: Restructuring for caring and effective education: piecing the puzzle together. 2 nd ed. Baltimore, Brooks. 2000, pp 93-128.

11) Kahn JM, Matthews FA, Angus DC, Barnato AE, Rubenfeld GD: Barriers to implementing the Leapfrog group recommendations for intensivist physician staffing: a survey of intensive care unit directors. J Crit Care 2007; 22: 97-103.

12) Koh Y: How to enhance critical care in Korea: challenges and vision. Korean J Crit Care Med 2014; 29: 246-9.

13) Pronovost PJ, Needham DM, Waters H, Birkmeyer CM, Calinawan JR Birkmeyer JD, et al: Intensive care unit physician staffing: financial modeling of the Leapfrog standard. Crit Care Med 2004; 32: 1247-53.

14) Moon YJ, Park JS, Ahn IW: Investigation on the management status of incentive pay system in hospital and strategy to invigorate. Korean J Health Service Management 2011; 5: 31-44..

15) Ward NS, Afessa B, Kleinpell R, Tisherman S, Ries $\mathrm{M}$, Howell M, et al: Intensivist/patient ratios in closed ICUs: a statement from the Society of Critical Care
Medicine Taskforce on ICU Staffing. Crit Care Med 2013; 41: 638-45.

16) Epstein AM: Public release of performance data: a progress report from the front. JAMA 2000; 283: 18846.

17) Millenson ML: Pay for performance: the best worst choice. Qual Saf Health Care 2004; 13: 323-4.

18) Shahian DM, Wolf RE, Iezzoni LI, Kirle L, Normand SL: Variability in the measurement of hospital-wide mortality rates. N Engl J Med 2010; 363: 2530-9.

19) Mackenzie SJ, Goldmann DA, Perla RJ, Parry GJ: Measuring hospital-wide mortality-pitfalls and potential. J Healthc Qual 2015 Jul 14 [Epub]. http://dx.doi. org/10.1111/jhq. 12080.

20) Pronovost PJ, Thompson DA, Holzmueller CG, Dorman T, Morlock LL: The organization of intensive care unit physician services. Crit Care Med 2007; 35: 225661.

21) Cho J, Lee HJ, Hong SB, Suh GY, Park MS, Kim SC, et al. Structure of intensive care unit and clinical outcomes in critically ill patients with influenza A/H1N1 2009. Korean J Crit Care Med 2012; 27: 65-69.

22) Kim JH, Hong SK, Kim KC, Lee MG, Lee KM, Jung $\mathrm{SS}$, et al. Influence of full-time intensivist and the nurse-to-patient ratio on the implementation of severe sepsis bundles in Korean intensive care units. J Crit Care 2012; 27: 414.e11-21.

23) Kim KD, Lee JW, Park HK. Feasibility and early outcomes of intensivist-led critical care after major trauma in the Korean ICU. Yonsei Med J 2013; 54: 432-6.

24) Park CM, Chun HK, Lee DS, Jeon K, Suh GY, Jeong JC. Impact of a surgical intensivist on the clinical outcomes of patients admitted to a surgical intensive care unit. Ann Surg Treat Res 2014; 86: 319-24.

25) OECD. OECD Health Care Quality Review: Korea Assessment and recommendations. [accessed on 9 July 2015]. Available at http://www.oecd.org/els/healthsystems/49818570.pdf.

26) Kellerman SE, Herold J. Physician response to surveys. A review of the literature. Am J Prev Med 2001; 20: 61-7.

27) Garland A, Gershengorn HB. Staffing in ICUs: physicians and alternative staffing models. Chest 2013; 143: 214-21. 